\title{
Beeinträchtigungen der sensomotorischen Kontrolle bei funktioneller Sprunggelenkinstabilität
}

\author{
Sensorimotor Deficits in Functional Ankle Instability
}

Autoren

Institut

\section{S. Steib, K. Pfeifer}

Institut für Sportwissenschaft und Sport, Friedrich-Alexander-Universität Erlangen-Nürnberg, Erlangen
Schlüsselwörter

- chronische Sprunggelenkinstabilität

○ neuromuskuläre Kontrolle

- Propriozeption

- posturale Kontrolle

- Sportverletzungen

Key words

- neuromuscular control

- proprioception

- postural control

- sports injuries
- chronic ankle instability

\section{Zusammenfassung \\ $\nabla$}

Distorsionen des oberen Sprunggelenks stellen mit einer Prävalenz von 25-30\% aller Sportunfälle neben dem Kniegelenk die am häufigsten betroffene Körperregion dar. Mindestens ein Drittel aller Betroffenen entwickelt eine chronische Gelenkinstabilität mit anhaltenden Beschwerden, die jedoch in vielen Fällen nicht auf eine mechanische Insuffizienz des Kapsel-Band-Apparats zurückzuführen ist. Hierfür wurde der Begriff der funktionellen Sprunggelenkinstabilität geprägt. Als Ursache werden Störungen des sensomotorischen Systems vermutet. Dazu zählen u.a. Beeinträchtigungen der Propriozeption, der posturalen Kontrolle sowie der Kraft und Reflexaktivität der gelenkumgebenden Muskulatur. Im vorliegenden Übersichtsbeitrag wird die aktuelle Studienlage hinsichtlich sensomotorischer Defizite bei funktioneller Sprunggelenkinstabilität aufgearbeitet. Die Ergebnisse werden vor dem Hintergrund neuerer neurophysiologischer Modelle zur Entstehung von Gelenkinstabilitäten diskutiert.

\section{Einleitung}

\section{Bibliografie}

Dol http://dx.doi.org/ 10.1055/s-0034-1396293

Online-publiziert 08.05.2015

Z Orthop Unfall 2015; 153 :

253-258 (c) Georg Thieme

Verlag KG Stuttgart · New York . ISSN 1864-6697

\section{Korrespondenzadresse}

Dr. Simon Steib

Institut für Sportwissenschaft und Sport

Friedrich-Alexander-Universität Erlangen-Nürnberg

Gebbertstraße 123b

91058 Erlangen

Tel.: $09131 / 8528178$

Fax: $09131 / 8528198$

simon.steib@fau.de

\section{$\nabla$}

\section{Epidemiologie}

Verletzungen des Sprunggelenks stellen mit einer Prävalenz von $25-30 \%$ aller Sportunfälle neben dem Kniegelenk die am häufigsten betroffene Körperregion dar [1]. Vor allem Distorsionen des oberen Sprunggelenks machen mit etwa 85\% den Großteil aller Sprunggelenkverletzungen aus [2]. Nur schätzungsweise die Hälfte aller am Sprunggelenk verletzten Sportler begibt sich jedoch in medizinische Behandlung [3]. Viele Verletzungen bleiben somit undokumentiert, weshalb die tatsächliche Prävalenz wohl meist unterschätzt wird. Die Verletzungsraten variieren zwischen den Disziplinen stark und sind in Sportarten, in denen vermehrt Gegnerkontakt, Sprünge und schnelle

\section{Abstract \\ $\nabla$}

Lateral ankle sprains are among the most common sports injuries, with a prevalence of 25 to $30 \%$ of all injuries. At least one-third of individuals develop long-term complaints and chronic instabilities at the ankle, which in many cases cannot be attributed to mechanical insufficiencies of the joint. This condition is referred to as functional ankle instability (FAI). Impairments of the sensorimotor control system, such as disturbed proprioception and postural control, as well as reduced muscle strength and reflex activity, have been suggested to contribute to the aetiology of FAI. This review summarises the current body of literature regarding sensorimotor control in individuals with FAI. We discuss the results in the context of current neurophysiological models of the development of functional joint instabilities.

Richtungswechsel auftreten, am höchsten [4]. Demzufolge zählen vor allem die Ballsportarten zu den Disziplinen mit der höchsten Prävalenz [1].

\section{Pathophysiologie und klinische Symptomatik}

Der typische Verletzungsmechanismus einer Sprunggelenkdistorsion ist das Supinationstrauma, auch als Inversionstrauma bezeichnet [5]. Je nach Schweregrad des Traumas ist eine Dehnung bzw. eine teilweise oder vollständige Ruptur der kapsulären und ligamentären Gelenkstrukturen die Folge. In den meisten Fällen ist der laterale Bandapparat des Sprunggelenks betroffen [6]. Unabhängig vom Schweregrad der initialen Verletzung entwickelt mindestens ein Drittel aller Betroffenen langfristige Beschwerden und Funk- 


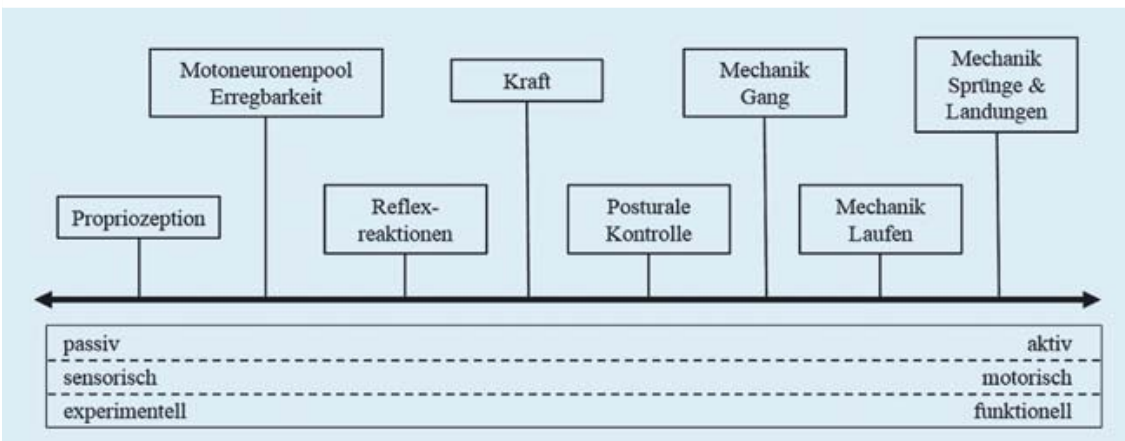

Abb. 1 Spektrum sensomotorischer Messungen in Verbindung mit funktioneller Sprunggelenkinstabilität (modifiziert nach Hertel [12]). $\leftrightarrow=$ Einordnung der diagnostischen Verfahren auf dem Kontinuum zwischen der Erfassung einzelner physiologischer Funktionssysteme (links) und der motorisch-funktionellen Leistung (rechts).

tionseinschränkungen [8], obwohl die akute klinische Symptomatik i.d.R. innerhalb weniger Tage bis Wochen abklingt [7]. Hierfür wurde der Begriff der „chronischen Sprunggelenkinstabilität“ (engl. „chronic ankle instability“) eingeführt [9]. Neben langfristig anhaltenden Beschwerden kommt es bei einer großen Zahl der Patienten zu wiederholten Verletzungsepisoden $[6,10]$. Das Risiko einer Wiederverletzung ist im Vergleich zu einer erstmaligen Distorsion um das bis zu 5-Fache erhöht [3].

Da die Instabilität in vielen Fällen nicht auf eine mechanische Insuffizienz des Sprunggelenks, also eine Gelenkbeweglichkeit über das normale physiologische Bewegungsausmaß hinaus, zurückzuführen ist, wird häufig zwischen mechanischer und funktioneller Sprunggelenkinstabilität unterschieden. Obwohl für die funktionelle Sprunggelenkinstabilität eine einheitliche Begriffsdefinition bislang fehlt [11] werden darunter meist anhaltende Beschwerden (Funktionseinschränkung, Schmerz, Schwächegefühl) und eine subjektiv wahrgenommene Instabilität am Sprunggelenk bei wiederholt auftretenden Verletzungs- oder Umknickepisoden (engl. „giving way“) zusammengefasst. In der vorliegenden Arbeit wird die funktionelle Sprunggelenkinstabilität verstanden als subjektiv empfundene Instabilität am Sprunggelenk bei fehlender mechanischer Insuffizienz, welche mit Funktionseinschränkungen und wiederholt auftretendem Umknicken einhergeht. Da mechanische Insuffizienzen meist nur operativ behandelt werden können und in vielen Fällen nicht die Ursache der chronischen Instabilität sind, stellen vor allem Studien zur Identifikation funktioneller Insuffizienzen und zur Erforschung ihrer Genese einen Schwerpunkt sportmedizinischer Forschungsanstrengungen dar. Die funktionelle Instabilität wird dabei vor allem mit Beeinträchtigungen des sensomotorischen Systems in Verbindung gebracht $[6,12]$.

\section{Evidenz sensomotorischer Defizite}

\section{$\nabla$}

Zur Einschätzung sensomotorischer Defizite bei funktioneller Sprunggelenkinstabilität liegen zahlreiche Studien vor. ๑ Abb. 1 zeigt das Spektrum der in der Literatur untersuchten Aspekte der sensomotorischen Kontrolle [12]. Im Folgenden soll die Evidenz hinsichtlich dieser verschiedenen Dimensionen aufgearbeitet und ihre Relevanz für die Entwicklung einer funktionellen Gelenkinstabilität diskutiert werden.

Aufgrund der in der Literatur häufig synonymen Verwendung der Begriffe „funktionelle“ bzw. „chronische“ Sprunggelenkinstabilität [11] ist eine eindeutige Abgrenzung der verschiedenen Studien und Probandenkollektive nicht in allen Fällen möglich. Daher wurden in der vorliegenden Literaturübersicht alle Studien berücksichtigt, deren Stichprobencharakteristika den o.g. Kriterien einer funktionellen Sprunggelenkinstabilität entsprechen.

\section{Propriozeption}

Die Propriozeption gehört zu den am intensivsten beforschten Komponenten der sensomotorischen Kontrolle bei funktioneller Sprunggelenkinstabilität. Der Gelenkstellungssinn wird dabei besonders häufig untersucht, zumeist operationalisiert über die aktive oder passive Reproduktion bestimmter Gelenkstellungen (Winkelreproduktionstests). Daneben zählen die Kinästhesie, erfasst durch Detektion langsamer passiver Gelenkbewegungen, und der Kraftsinn, erhoben mittels Kraftreproduktionsaufgaben, zu den analysierten Dimensionen der Propriozeption.

McKeon und McKeon [13] fassen in einer aktuellen Übersichtsarbeit 10 Studien metaanalytisch zusammen. Hierdurch konnten moderate Einschränkungen (standardisierte Mittelwertdifferenz $=0,50$, 95\%-Konfidenzintervall 0,36-0,64) des Gelenkstellungssinns bei funktioneller Sprunggelenkinstabilität nachgewiesen werden. Dennoch bestehen aufgrund der Heterogenität der Studienpopulationen und der niedrigen methodischen Qualität zahlreicher Studien Unsicherheiten bez. der Größe und klinischen Bedeutsamkeit der Defizite [13,14].

Die wenigen zur Einschätzung kinästhetischer Defizite verfügbaren Untersuchungen kommen zu widersprüchlichen Ergebnissen. Refshauge et al. [15] konnten eine erhöhte Wahrnehmungsschwelle passiver Gelenkbewegungen bei Patienten mit funktioneller Sprunggelenkinstabilität nachweisen. Die gleiche Autorengruppe fand jedoch in 2 anderen Untersuchungen keine Unterschiede zu Personen mit stabilen Gelenken $[16,17]$. Weitere Studien stellten bei Probanden mit einseitiger Instabilität im Seitenvergleich kinästhetische Defizite am instabilen Sprunggelenk fest $[18,19]$, während Hubbard und Kaminsky [20] keine Differenzen im Seitenvergleich verifizieren konnten.

Zur Untersuchung des Kraftsinns bei Patienten mit funktioneller Sprunggelenkinstabilität liegen ebenfalls nur wenige Arbeiten vor [21-24]. Diese konzentrieren sich auf Kraftreproduktionsaufgaben der Peronealmuskulatur. Defizite konnten sowohl intraindividuell, also zwischen dem betroffenen und asymptomatischen Gelenk [21,22], als auch im Vergleich zu gesunden Kontrollprobanden $[23,24]$ nachgewiesen werden. Eine aussagekräftige Abschätzung der klinischen Bedeutsamkeit dieser Ergebnisse ist aufgrund der geringen Datenlage jedoch kaum möglich. Weiterhin fehlen bislang Untersuchungen an anderen Muskelgruppen des Sprunggelenks.

\section{Posturale Kontrolle}

Die am häufigsten untersuchte Zielgröße zur Beurteilung der Funktionsfähigkeit des sensomotorischen Systems stellt die posturale Kontrolle dar. Der Großteil dieser Studien misst hierzu die statische Haltungskontrolle im Einbeinstand, in jüngerer Vergangenheit wurde vermehrt auch die dynamische posturale Kontrolle untersucht. Die Ergebnisse dieser Forschungsarbeiten wurden 
in jüngerer Vergangenheit mehrfach metaanalytisch zusammengefasst [14,25-27]. Übereinstimmend wurden bei Personen mit funktioneller Sprunggelenkinstabilität Beeinträchtigungen der statischen und dynamischen posturalen Kontrolle nachgewiesen, die berichteten Effektstärken sind als klein bis moderat einzuschätzen (standardisierte Mittelwertdifferenz 0,3-0,6). Defizite der statischen Haltungskontrolle treten besonders unter erhöhten sensorischen Anforderungen (geschlossene Augen, instabiler Untergrund) hervor [14]. Bislang besteht jedoch kein Konsens bez. der Messverfahren mit dem höchsten diagnostischen Wert zur Identifikation von Defiziten der Haltungskontrolle [25, $28,29]$.

\section{Neuromuskuläre Kontrolle}

Zur Einschätzung der Schutzfunktion der sprunggelenkstabilisierenden Muskulatur wird häufig die Reflexaktivität der Mm. peroneus longus und brevis und des M. tibialis anterior bei unvorhersehbarer Sprunggelenkinversion untersucht. Trotz des Vorliegens einer beträchtlichen Anzahl an Studien konnte in aktuellen Metaanalysen kein eindeutiger Nachweis für Defizite in der Reaktionszeit der Peronealmuskulatur erbracht werden $[14,26]$.

Eine verminderte willkürliche Aktivierbarkeit der Sprunggelenkmuskulatur - auch als arthrogene Muskelhemmung (engl. „arthrogenic muscle inhibition“) bezeichnet - bei funktioneller Sprunggelenkinstabilität konnte in 2 Arbeiten belegt werden $[30,31]$. Neben einer eingeschränkten Innervierbarkeit distaler Muskelgruppen (Mm. peronei) wiesen Sedory et al. [32] auch eine Hemmung proximaler Muskeln (Mm. ischiocrurale) bei Personen mit funktioneller Sprunggelenkinstabilität nach.

\section{Kraftfähigkeiten}

Die Kraftfähigkeiten der sprunggelenkumgebenden Muskulatur wurden vorwiegend in Sprunggelenkinversion und -eversion untersucht. Zu den Zielgrößen gehören die isometrische, konzentrische und exzentrische Maximalkraft sowie das Verhältnis der Kraftwerte von Evertoren zu Invertoren. Trotz zahlreicher Untersuchungen ist die Datenlage hierzu bislang sehr uneinheitlich, weshalb das Vorliegen von Kraftdefiziten in der Literatur bis heute kontrovers diskutiert wird. Arnold et al. [33] fassen in ihrer Metaanalyse 12 Studien zu konzentrischen Kraftfähigkeiten der Mm. peronei bei funktioneller Sprunggelenkinstabilität zusammen und belegen ein signifikantes Kraftdefizit von jedoch kleiner Effektstärke (standardisierte Mittelwertdifferenz $=0,22$; 95\%Konfidenzintervall 0,12-0,33). Hiller et al. [14] stellen in ihrer Metaanalyse hingegen signifikante Effekte für die konzentrischen Kraftfähigkeiten der Invertoren, nicht aber der Evertoren des Sprunggelenks fest. Nur wenige Studien gehen auf die Kraft der Flexoren und Extensoren des Sprunggelenks ein. Fox et al. [34] und Gribble et al. [35] konnten Defizite der Plantarflexoren des Sprunggelenks, nicht jedoch der Dorsalextensoren, bestätigen.

\section{Bewegungsmuster}

Studien zur Untersuchung beeinträchtigter Bewegungsmuster bei funktioneller Sprunggelenkinstabilität beinhalten zumeist kinematische Analysen des Sprunggelenks beim Gehen bzw. bei Sprunglandungen. So konnten Spaulding et al. [36] während des initialen Fersenkontakts beim Gehen auf ebener Fläche eine erhöhte Plantarflexion bei instabilen im Vergleich zu gesunden Sprunggelenken belegen. Außerdem wurde eine erhöhte Inversion des Sprunggelenks kurz vor und während des Fußaufsatzes gezeigt [37,38]. Selbiges stellten Delahunt et al. [39] bei Personen mit funktioneller Sprunggelenkinstabilität auch zum Zeitpunkt
Tab. 1 Sensomotorische Defizite bei funktioneller Sprunggelenkinstabilität.

\begin{tabular}{|c|c|c|c|}
\hline Defizit & & Assessment & $\begin{array}{l}\text { Daten- } \\
\text { lage }\end{array}$ \\
\hline \multirow[t]{3}{*}{$\begin{array}{l}\text { Proprio- } \\
\text { zeption }\end{array}$} & $\begin{array}{l}\text { Gelenkstel- } \\
\text { lungssinn }\end{array}$ & $\begin{array}{l}\text { Winkelreproduktionstests } \\
\text { (aktiv/passiv) }\end{array}$ & $\uparrow \uparrow \uparrow$ \\
\hline & Kinästhesie & $\begin{array}{l}\text { Erkennung langsamer passiver } \\
\text { Gelenkbewegungen }\end{array}$ & $\leftrightarrow$ \\
\hline & Kraftsinn & Kraftreproduktionsaufgaben & $\uparrow$ \\
\hline \multirow{2}{*}{$\begin{array}{l}\text { posturale } \\
\text { Kontrolle }\end{array}$} & statisch & Posturografie im Einbeinstand & $\uparrow \uparrow \uparrow$ \\
\hline & dynamisch & $\begin{array}{l}\text { Star Excursion Balance Test } \\
\text { Time to Stabilization } \\
\text { Dynamic Postural Stability Index } \\
\text { Multiple Hop Test }\end{array}$ & $\uparrow \uparrow \uparrow$ \\
\hline \multirow[t]{2}{*}{$\begin{array}{l}\text { neuro- } \\
\text { muskuläre } \\
\text { Kontrolle }\end{array}$} & $\begin{array}{l}\text { Muskel- } \\
\text { reaktions- } \\
\text { zeit }\end{array}$ & $\begin{array}{l}\text { Muskelaktivität bei plötzlicher } \\
\text { Sprunggelenkinversion } \\
\text { (Elektromyografie) }\end{array}$ & $\leftrightarrow$ \\
\hline & $\begin{array}{l}\text { arthrogene } \\
\text { Muskel- } \\
\text { hemmung }\end{array}$ & H-Reflex-Messung & $\uparrow$ \\
\hline \multirow[t]{2}{*}{$\begin{array}{l}\text { Muskel- } \\
\text { kraft }\end{array}$} & $\begin{array}{l}\text { Evertoren, } \\
\text { Invertoren }\end{array}$ & $\begin{array}{l}\text { isometrische, exzentrische und } \\
\text { konzentrische Kraftmessungen }\end{array}$ & $\leftrightarrow$ \\
\hline & $\begin{array}{l}\text { Plantarflexo- } \\
\text { ren, Dorsal- } \\
\text { extensoren }\end{array}$ & & $\leftrightarrow$ \\
\hline $\begin{array}{l}\text { Bewegungs- } \\
\text { muster }\end{array}$ & $\begin{array}{l}\text { Sprungge- } \\
\text { lenkkine- } \\
\text { matik }\end{array}$ & $\begin{array}{l}\text { kinematische Bewegungsana- } \\
\text { lyse (u. a. Gang, Laufen, Sprung- } \\
\text { landungen) }\end{array}$ & $\uparrow \uparrow$ \\
\hline
\end{tabular}

$\uparrow \uparrow \uparrow=$ Nachweis durch systematische Reviews oder Metaanalysen; $\uparrow \uparrow=$ bestätigt durch konsistente Ergebnisse mehrerer Studien; $\uparrow$ = wenige Studien mit jedoch konsistenten Ergebnissen; $\leftrightarrow$ = widersprüchliche Studienlage

des Bodenkontakts bei Sprunglandungen fest. Caulfield und Garrett [40] konnten zudem eine erhöhte Dorsalextension am Sprunggelenk und eine erhöhte Knieflexion direkt vor und nach einer einbeinigen Sprunglandung nachweisen.

\section{Zusammenfassung}

Eine zusammenfassende Darstellung der oben beschriebenen Studienlage ist in $\odot$ Tab. 1 gegeben. Beeinträchtigungen konnten auf verschiedenen Ebenen des sensomotorischen Systems festgestellt werden. Dennoch besteht Ungewissheit hinsichtlich der tatsächlichen Existenz bzw. exakten Lokalisation und des Ausmaßes der Defizite.

\section{Diskussion}

Freeman und Kollegen [41] brachten die Ätiologie der funktionellen Instabilität des Sprunggelenks erstmals mit Defiziten der sensomotorischen Kontrolle in Verbindung. Sie berücksichtigten dabei ausschließlich Feedbackmechanismen und vermuteten die primäre Ursache in einer "Gelenk-Deafferenzierung“ [41], also einem reduzierten afferenten Input von Mechanorezeptoren bedingt durch strukturelle Schädigung passiver Gelenkstrukturen. Das daraus resultierende „propriozeptive Defizit“ und die damit verbundene Störung der reflektorischen muskulären Gelenkkontrolle wurde als Ursache wiederholter Instabilitätsmomente und Verletzungsepisoden postuliert [41]. Nachdem Freemans Theorie lange Jahre weitgehend unbestritten blieb, haben neuere Erkenntnisse zu einer Erweiterung des Ursachenkomplexes geführt. 


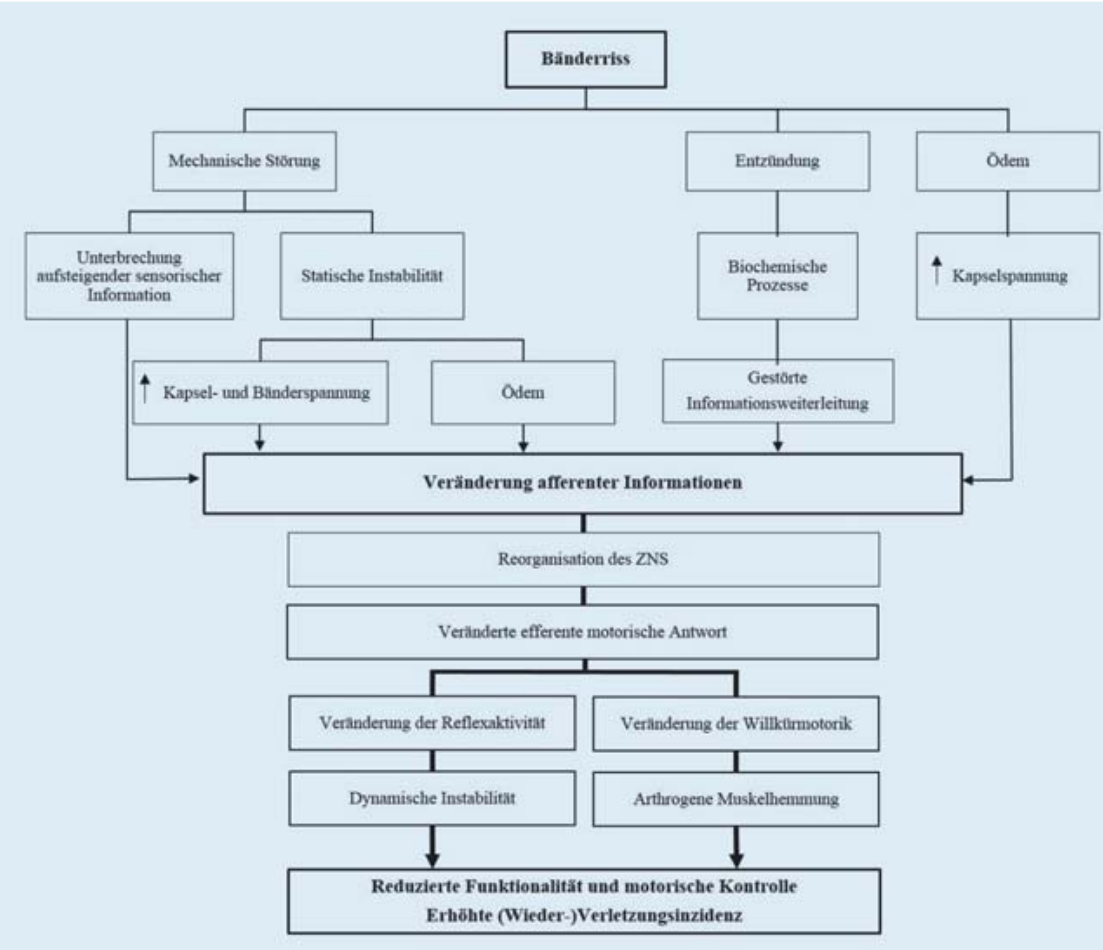

Abb. 2 Hypothetische Entstehung der funktionellen Gelenkstabilität durch verletzungsbedingte Feedback- und Feedforwardmechanismen (modifiziert nach [49]).

\section{Die Bedeutung der Propriozeption}

Zwar wurden Einschränkungen der Propriozeption im Rahmen aktueller messmethodischer Möglichkeiten bei funktioneller Sprunggelenkinstabilität dargestellt $[12,13]$, was eine Beeinträchtigung von Mechanorezeptoren durch Kapsel-Band-Verletzungen im Sprunggelenk vermuten lässt [12]. Allerdings ist die funktionelle Bedeutung des Verlusts dieser Gelenkafferenzen, besonders bezogen auf reine Feedbackmechanismen, fraglich [42]. Dies untermauern Arbeiten, die bei lokaler Anästhesie des lateralen Bandapparats bzw. des gesamten oberen Sprunggelenks keine negativen Effekte auf Muskelreflexaktivitäten am Sprunggelenk oder die posturale Kontrolle feststellen konnten $[43,44]$. Mechanorezeptoren in den Bänderstrukturen sind nur Teil eines Komplexes zahlreicher Rezeptorpopulationen mit teilweise redundanter Funktionsweise. Eine Kompensation des Verlusts von Rezeptoren ist daher wahrscheinlich [42]. Demzufolge würde die Schädigung von Gelenksensoren nicht zwingend in einer Beeinträchtigung von Feedbackmechanismen resultieren, die an der Haltungskontrolle und reflektorischen Muskelaktivierung beteiligt sind.

\section{Spinale und supraspinale Mechanismen}

Vielmehr werden veränderte Gelenkafferenzen als Ursache spinaler und supraspinaler Reorganisationsprozesse vermutet [12]. Diese Annahme wird durch empirische Befunde gestützt, die bei Patienten mit funktioneller Sprunggelenkinstabilität eine Hemmung der Alpha-Motoneuronen-Aktivierbarkeit proximaler [32] und distaler [30,31] Muskelgruppen der unteren Extremität darstellen. Weiterhin konnten Sefton et al. [45] prä- und postsynaptische Modulationen spinaler Reflexe darstellen. Diese Ergebnisse legen somit Adaptationen auf spinaler Ebene nahe.

Die Annahme einer verletzungsbedingten Reorganisation auf supraspinaler Ebene wird von hier dargestellten Arbeiten gestützt, die Veränderung von Bewegungsmustern und Muskelaktivierungsmustern bei Personen mit funktioneller Sprunggelenkinsta- bilität nachweisen konnten $[37,39,46]$. Weiterhin spricht auch das Vorliegen bilateraler Defizite der sensomotorischen Kontrolle bei einseitiger Sprunggelenkinstabilität für zentrale Reorganisationen [12,27,47]. Vergleichbare Befunde bei Patienten nach Ruptur des vorderen Kreuzbands stützen ebenfalls die Annahme supraspinaler Adaptationsprozesse infolge einer Bänderverletzung. So konnten beispielsweise Kapreli et al. [48] bei dieser Patientengruppe den Nachweis von Reorganisationen in mehreren Kortexarealen erbringen.

\section{Reorganisationsprozesse des sensomotorischen Systems} Gemeinsam sprechen die dargestellten Studienergebnisse für eine Störung multipler Feedforward- und Feedbackprozesse auf verschiedenen Ebenen des sensomotorischen Systems. Kapreli und Athanasopoulos [49] entwickelten am Beispiel der vorderen Kreuzbandruptur ein hypothetisches Modell für verletzungsbedingte Reorganisationsprozesse des zentralen Nervensystems und die daraus resultierende Genese einer funktionellen Gelenkinstabilität ( $\odot$ Abb. 2).

Sie postulieren, dass gestörte somatosensorische Informationen des betroffenen Gelenks Reorganisationsprozesse im ZNS induzieren, welche langfristig zu einer Reduktion der efferenten Kontrolle der gelenkstabilisierenden Muskulatur führen. Spezifisch verursachen veränderte Gelenkafferenzen über das fusimotorische System eine Beeinträchtigung der Muskelspindelaktivität, wodurch die situationsangepasste Stiffness-Regulation und die Reflexaktivität der Muskulatur eingeschränkt sind. Aufgrund der verminderten Erregbarkeit des Alpha-Motoneuronenpools der Gelenkmuskulatur, der reduzierten antizipatorischen und der verzögerten reflektorischen Muskelaktivität verliert die Muskulatur ihre dynamische Schutzfunktion. Modifizierte Bewegungsmuster und eine verschlechterte posturale Kontrolle erhöhen zudem die Wahrscheinlichkeit ungünstiger Gelenkbelastungen und Fehlpositionierungen. Gemeinsam bieten diese Mechanismen einen Erklärungsansatz für die reduzierte Funktionsfähigkeit, die 
häufig auftretenden Instabilitätsmomente und die hohen Wiederverletzungsraten.

\section{Schlussfolgerung}

Die funktionelle Sprunggelenkinstabilität stellt ein komplexes Beschwerdebild dar, welches sich in chronischen Veränderungen auf verschiedenen Ebenen des sensomotorischen Systems manifestiert. Die Untersuchung der zugrunde liegenden Mechanismen und der Bedeutung dieser bleibenden Reorganisationen für die Entwicklung von Instabilitäten und wiederholten Verletzungsepisoden stellt eine Herausforderung für zukünftige Forschungsanstrengungen dar. Aus praktischer Sicht sprechen die hier dargestellten Befunde für eine langfristige, multimodale Therapie von Sprunggelenkverletzungen. Neben der Verbesserung der Gelenkfunktion und Schmerzsituation stehen dabei vor allem die Verbesserung der Bewegungsqualität und aktiven (muskulären) Gelenkstabilisierung im Vordergrund, was letztendlich repetitive Verletzungsepisoden verhindern soll. Hierbei sind konservative Behandlungskonzepte operativen Eingriffen vorzuziehen. Vor allem bewegungs- und physiotherapeutischen Maßnahmen kommt dabei eine große Bedeutung zu. Häufig eingesetzte Therapiekonzepte beinhalten Balanceübungen auf stabilen und instabilen Unterlagen, oft kombiniert mit Krafttraining, Sprungübungen oder sportartspezifischen Übungsinhalten. Diese Programme werden meist als sensomotorisches, propriozeptives oder auch neuromuskuläres Training bezeichnet und ihre Wirksamkeit in der Rehabilitation von Sportverletzungen ist nachgewiesen [50]. Für die Behandlung der funktionellen Sprunggelenkinstabilität gilt es, in zukünftigen Arbeiten die wichtigsten Therapieinhalte und Trainingsmodalitäten (z.B. Dauer, Häufigkeit) herauszuarbeiten.

\section{Interessenkonflikt: Nein}

\section{Literaturverzeichnis}

1 Fong $D$, Hong $Y$, Chan $L$ et al. A systematic review on ankle injury and ankle sprain in sports. Sports Med 2007; 37: 73-94

2 Steinbrück K. Epidemiologie von Sportverletzungen - 25-Jahres-Analyse einer sportorthopädisch-traumatologischen Ambulanz. Sportverletz Sportschaden 1999; 13: 38-52

3 McKay GD, Goldie PA, Payne WR et al. Ankle injuries in basketball: injury rate and risk factors. Br J Sports Med 2001; 35: 103-108

4 Nelson AJ, Collins CL, Yard EE et al. Ankle injuries among United States high school sports athletes, 2005-2006. J Athl Train 2007; 42: 381-387

5 Lohrer H, Alt W, Gollhofer A et al. Verletzungen am lateralen Kapselbandapparat des Sprunggelenks - eine Übersicht. Dtsch Z Sportmed 2000; 51: 196-203

6 Hertel J. Functional anatomy, pathomechanics, and pathophysiology of lateral ankle instability. J Athl Train 2002; 37: 364-375

7 van Rijn RM, van Os AG, Bernsen RMD et al. What is the clinical course of acute ankle sprains? A systematic literature review. Am J Med 2008; 121: $324-331$

8 Anandacoomarasamy A, Barnsley L. Long term outcomes of inversion ankle injuries. Br J Sports Med 2005; 39: e14

9 Valderrabano V, Leumann A, Pagenstert G et al. Chronische Instabilität des oberen Sprunggelenks im Sport: Ein Review für Sportärzte. Sportverletz Sportschaden 2006; 20: 177-183

10 Hiller CE, Kilbreath SL, Refshauge KM. Chronic ankle instability: evolution of the model. J Athl Train 2011; 46: 133-141

11 Delahunt E, Coughlan GF, Caulfield B et al. Inclusion criteria when investigating insufficiencies in chronic ankle instability. Med Sci Sports Exerc 2010; 42: 2106-2121

12 Hertel J. Sensorimotor deficits with ankle sprains and chronic ankle instability. Clin Sports Med 2008; 27: 353-370
13 McKeon JMM, McKeon PO. Evaluation of joint position recognition measurement variables associated with chronic ankle instability: a metaanalysis. J Athl Train 2012; 47: 444-456

14 Hiller CE, Nightingale EJ, Lin CC et al. Characteristics of people with recurrent ankle sprains: a systematic review with meta-analysis. $\mathrm{Br} \mathrm{J}$ Sports Med 2011; 45: 660-672

15 Refshauge KM, Kilbreath SL, Raymond J. Deficits in detection of inversion and eversion movements among subjects with recurrent ankle sprains. J Orthop Sports Phys Ther 2003; 33: 166-173

16 de Noronha M, Refshauge KM, Kilbreath SL et al. Loss of proprioception or motor control is not related to functional ankle instability: an observational study. Aust J Physiother 2007; 53: 193-198

17 Refshauge KM, Kilbreath SL, Raymond J. The effect of recurrent ankle inversion sprain and taping on proprioception at the ankle. Med Sci Sports Exerc 2000; 32: 10-15

18 Forkin DM, Koczur C, Battle R et al. Evaluation of kinesthetic deficits indicative of balance control in gymnasts with unilateral chronic ankle sprains. J Orthop Sports Phys Ther 1996; 23: 245-250

19 Lentell G, Baas B, Lopez D et al. The contributions of proprioceptive deficits, muscle function, and anatomic laxity to functional instability of the ankle. J Orthop Sports Phys Ther 1995; 21: 206-215

20 Hubbard TJ, Kaminski TW. Kinesthesia is not affected by functional ankle instability status. J Athl Train 2002; 37: 481-486

21 Arnold BL, Docherty CL. Low-load eversion force sense, self-reported ankle instability, and frequency of giving way. J Athl Train 2006; 41: 233-238

22 Docherty CL, Arnold BL, Hurwitz S. Contralateral force sense deficits are related to the presence of functional ankle instability. J Orthop Res 2006; 24: 1412-1419

23 Docherty CL, Arnold BL. Force sense deficits in functionally unstable ankles. J Orthop Res 2008; 26: 1489-1493

24 Wright CJ, Arnold BL. Fatigue's effect on eversion force sense in individuals with and without functional ankle instability. J Sport Rehabil 2012; 21: 127-136

25 Arnold BL, de la Motte S, Linens $S$ et al. Ankle instability is associated with balance impairments: a meta-analysis. Med Sci Sports Exerc 2009; 41: 1048-1062

26 Munn J, Sullivan SJ, Schneiders AG. Evidence of sensorimotor deficits in functional ankle instability: a systematic review with meta-analysis. J Sci Med Sport 2010; 13: 2-12

27 Wikstrom EA, Naik S, Lodha $N$ et al. Bilateral balance impairments after lateral ankle trauma: a systematic review and meta-analysis. Gait Posture 2010; 31: 407-414

28 Ross SE, Guskiewicz KM, Gross MT et al. Balance measures for discriminating between functionally unstable and stable ankles. Med Sci Sports Exerc 2009; 41: 399-407

29 Wikstrom EA, Fournier KA, McKeon PO. Postural control differs between those with and without chronic ankle instability. Gait Posture 2010; 32: $82-86$

30 McVey ED, Palmieri RM, Docherty CL et al. Arthrogenic muscle inhibition in the leg muscles of subjects exhibiting functional ankle instability. Foot Ankle Int 2005; 26: 1055-1061

31 Palmieri-Smith RM, Hopkins JT, Brown TN. Peroneal activation deficits in persons with functional ankle instability. Am J Sports Med 2009; 37: 982-988

32 Sedory EJ, McVey ED, Cross KM et al. Arthrogenic muscle response of the quadriceps and hamstrings with chronic ankle instability. J Athl Train 2007; 42: 355-360

33 Arnold BL, Linens SW, de la Motte SJ et al. Concentric evertor strength differences and functional ankle instability: a meta-analysis. J Athl Train 2009; 44: 653-662

34 Fox J, Docherty CL, Schrader J et al. Eccentric plantar-flexor torque deficits in participants with functional ankle instability. J Athl Train 2008; 43: $51-54$

35 Gribble PA, Robinson RH. An examination of ankle, knee, and hip torque production in individuals with chronic ankle instability. J Strength Cond Res 2009; 23: 395-400

36 Spaulding SJ, Livingston LA, Hartsell HD. The influence of external orthotic support on the adaptive gait characteristics of individuals with chronically unstable ankles. Gait Posture 2003; 17: 152-158

37 Delahunt E, Monaghan K, Caulfield B. Altered neuromuscular control and ankle joint kinematics during walking in subjects with functional instability of the ankle joint. Am J Sports Med 2006; 34: 1970-1976 
38 Monaghan K, Delahunt E, Caulfield B. Ankle function during gait in patients with chronic ankle instability compared to controls. Clin Biomech (Bristol, Avon) 2006; 21: 168-174

39 Delahunt E, Monaghan K, Caulfield B. Changes in lower limb kinematics, kinetics, and muscle activity in subjects with functional instability of the ankle joint during a single leg drop jump. J Orthop Res 2006; 24 : 1991-2000

40 Caulfield BM, Garrett M. Functional instability of the ankle: differences in patterns of ankle and knee movement prior to and post landing in a single leg jump. Int J Sports Med 2002; 23: 64-68

41 Freeman MA, Dean MR, Hanham IW. The etiology and prevention of functional instability of the foot. J Bone Joint Surg Br 1965; 47: 678685

42 Hogervorst T, Brand RA. Mechanoreceptors in joint function. J Bone Joint Surg Am 1998; 80: 1365-1378

43 Konradsen L, Ravn JB, Sørensen AI. Proprioception at the ankle: the effect of anaesthetic blockade of ligament receptors. J Bone Joint Surg Br 1993; 75: 433-436
44 Riemann BL, Myers JB, Stone DA et al. Effect of lateral ankle ligament anesthesia on single-leg stance stability. Med Sci Sports Exerc 2004; 36: 388-396

45 Sefton JM, Hicks-Little CA, Hubbard TJ et al. Segmental spinal reflex adaptations associated with chronic ankle instability. Arch Phys Med Rehabil 2008; 89: 1991-1995

46 Delahunt E, Monaghan K, Caulfield B. Ankle function during hopping in subjects with functional instability of the ankle joint. Scand J Med Sci Sports 2007; 17: 641-648

47 McKeon PO, Hertel J. Systematic review of postural control and lateral ankle instability, Part I: Can deficits be detected with instrumented testing? J Athl Train 2008; 43: 293-304

48 Kapreli E, Athanasopoulos S, Gliatis J et al. Anterior cruciate ligament deficiency causes brain plasticity: a functional MRI study. Am J Sports Med 2009; 37: 2419-2426

49 Kapreli E, Athanasopoulos $S$. The anterior cruciate ligament deficiency as a model of brain plasticity. Med Hypotheses 2006; 67: 645-650

50 Zech A, Hübscher M, Vogt L et al. Neuromuscular training for rehabilitation of sports injuries: a systematic review. Med Sci Sports Exerc 2009; 41: $1831-1841$ 